\title{
Comparison of Autophagy mRNA Expression between Chronic Otitis Media With and Without Cholesteatoma
}

\author{
Junyang Jung ${ }^{1 *}$, Su Young Jung ${ }^{2 *}$, Myung Gu Kim³ , Young II Kim4, \\ Sang Hoon $\mathrm{Kim}^{5}$, and Seung Geun $\mathrm{YeO}^{4,5}$ \\ ${ }^{1}$ Departments of Anatomy and Neurobiology, ${ }^{5}$ Otorhinolaryngology, School of Medicine, KyungHee University, Seoul, Korea \\ ${ }^{2}$ Department of Otorhinolaryngology-Head and Neck Surgery, Myongji Hospital, Hanyang University College of Medicine, Goyang, Korea \\ ${ }^{3}$ Department of Otorhinolaryngology, Samsung Changwon Hospital, Sungkyunkwan University School of Medicine, Changwon, Korea \\ ${ }^{4}$ Medical Science Research Institute, KyungHee University Medical Center, Seoul, Korea
}

\begin{abstract}
Received March 6, 2020
Revised April 10,2020

Accepted April 30, 2020
\end{abstract}

\section{Address for correspondence \\ Seung Geun Yeo, MD, PhD \\ Department of Otohinolaryngology- \\ Head and Neck Surgery, \\ School of Medicine, \\ KyungHee University, \\ 23 Kyungheedae-ro, Dongdaemun-gu, \\ Seoul 02447, Korea \\ Tel +82-2-958-8474 \\ Fax +82-2-958-8470 \\ E-mail yeo2park@gmail.com}

*These authors contributed equally to this work.
Background and Objectives: Autophagy is known to be associated with pathogen infection However, the expression of autophagy-related proteins has not been studied in chronic otitis media without cholesteatoma (COM) or with cholesteatoma (CholeOM). This study aimed to determine whether there is a difference between COM and CholeOM in autophagy-related gene mRNA expression. Subjects and Methods: For 47 patients with chronic otitis media, the inflammatory tissues were classified into granulation tissue (COM) or cholesteatoma (CholeOM) according to biopsy results. Results: PI3K mRNA expression (COM vs. CholeOM, mean \pm SD, $0.009 \pm 0.010$ vs. $0.003 \pm 0.004 ; p=0.004$ ) was lower, whereas Beclin-1 mRNA expression ( $0.089 \pm 0.107$ vs. $0.176 \pm 0.163 ; p=0.034)$ was higher in the CholeOM group. Expression of PI3K mRNA in the CholeOM group was lower than that in the COM subgroups with presence of bacteria $(0.022 \pm 0.019$ vs. $0.001 \pm 0.001 ; p=0.001)$, otorrhea $(0.049 \pm 0.068$ vs. $0.003 \pm 0.004 ; p=0.004)$, and hearing loss over $40 \mathrm{~dB}(0.083 \pm 0.130$ vs . $0.003 \pm 0.004 ; p=0.005)$. Conclusions: The data suggested that different autophagy proteins play important roles in chronic otitis media according to the presence or absence of cholesteatoma.

J Audiol Otol 2020;24(4):191-197

KEY WORDS: Autophagy · Otitis media · Cholesteatoma.

\section{Introduction}

Autophagy is a general term for lysosome-based mechanisms that break down targets in the cytoplasm. Autophagy can effectively remove cytosolic infectious pathogens directly through the lysosome or enhance phagocytosis of extracellular pathogens through the cell membrane. Autophagy is activated by infection with bacteria [1,2].

Middle ear infection is a general term applied to all inflammatory reactions in the middle ear showing pathological changes that involve bone destruction of structures as well as changes in the middle ear cavity and middle ear mucosal, epithelial, and subepithelial cells [3]. The cause of otitis media is

This is an Open Access article distributed under the terms of the Creative Commons Attribution Non-Commercial License (https://creativecommons.org/licenses/by-nc/4.0/) which permits unrestricted non-commercial use, distribution, and reproduction in any medium, provided the original work is properly cited. complex and known to involve multiple factors, of which the most important are dysfunction of the eustachian tube and bacterial infection. After infection with bacteria, autophagy is activated and various immune responses are thought to be involved in the pathogenesis of chronic otitis media; however, not many studies have investigated the underlying mechanism.

A variety of different conditions and biochemical factors associated with the immune response are involved in various aspects of the development of inflammatory lesions in the middle ear cavity, and these processes are responsible for the recurrence and chronicity of otitis media [4-6]. The role of autophagy in chronic otitis media may be different depending on the presence or absence of accompanying cholesteatoma. It is likely that the mRNA expression of autophagy proteins differs according to clinical features and presence or absence of cholesteatoma, however, there is no reported study on this. Therefore, we investigated the expression pattern of 
autophagy-related mRNAs in chronic otitis media and compared expression levels according to the presence of cholesteatoma, bacterial infection, or otorrhea, as well as the degree of hearing loss.

\section{Subjects and Methods}

\section{Subjects}

The study population consisted of 47 patients who visited the Department of Otorhinolaryngology-Head and Neck clinic at a single tertiary medical center. Chronic otitis media was defined as the presence of persistent symptoms, such as hearing loss, otorrhea, tinnitus, and otalgia ( \pm perforation of the tympanic membrane \pm otorrhea \pm attic destruction) lasting longer than 3 months, with the identification of a lesion on the middle ear and mastoid process on temporal bone computed tomography (TBCT). The presence or absence of otorrhea was confirmed with otoscopy and endoscopy; pus culture was conducted if otorrhea was observed in the external ear canal or tympanic membrane of the middle ear. The presence and severity of hearing disturbance was assessed with pure tone audiometry (PTA), preoperative TBCT was performed to assess the presence and severity of middle-ear lesions, and inflammatory tissue was obtained for biopsy during surgery to confirm the diagnosis of chronic otitis media. Chronic inflammation, granulation tissue, and cholesteatoma were identified with histologic biopsy of the middle ear inflammatory tissue. Based on results of histologic biopsy, the chronic otitis media patients were divided into the chronic otitis media without cholesteatoma (COM) group (presence of chronic inflammation and granulation tissue without cholesteatoma) and the chronic otitis media with cholesteatoma (CholeOM) group (presence of cholesteatoma).

We received prior permission for use of patient samples with written informed consent from the patients or their parents or guardians, and explained the purpose of the experiment to them. Patients who were suspected of having acute otitis media, otitis media with effusion, head and neck anomalies, systemic diseases, or congenital or acquired immune deficiencies were excluded.

The study protocol was approved by the Institutional Review Board of KyungHee Medical Hospital (IRB 2017-12030).

\section{Bacterial culture}

When otorrhea was observed in patients, the sample was taken and tested for bacterial culture. After cleansing of the external auditory canal, the otorrhea sample was aseptically collected using sterilized cotton while employing an antisep- tic otoscope to prevent contact with the external auditory canal. All clinical samples were sampled using sterile cotton swabs (Xomed Trace Products, Jacksonville, FL, USA), which were immersed in Stuart transport medium. Solid blood agar and liquid thioglycolate medium (Hangang, Gun-po, Korea) were inoculated with these samples, and the cultures were incubated for $24 \mathrm{~h}$ at $35.8^{\circ} \mathrm{C}$. Bacteria that formed colonies were identified by Gram staining and biochemical testing.

\section{RNA extraction and real-time PCR procedures}

Total RNA was purified from patient samples using TRIzol solution (Invitrogen, Carlsbad, CA, USA) following the manufacturer's protocol. RNA was isolated from tissues using RNeasy Mini kit (Qiagen, Hilden, Germany). First-strand cDNA synthesis was performed with $1.5 \mu \mathrm{g}$ of total RNA, which was transcribed to cDNA using a reverse transcription system with random hexamers (Promega; Madison, WI, USA) according to the manufacturer's protocol. Real-time PCR was performed on a StepOnePlus real-time PCR system with Power SYBR Green PCR Master Mix (Applied Biosystems, Foster City, CA, USA). PCR was performed with $2 \mu \mathrm{L}$ of cDNA in a $20 \mu \mathrm{L}$ reaction mixture containing $10 \mu \mathrm{L}$ of Power SYBR Green PCR Master Mix, $2 \mu \mathrm{L}$ of primers, and $7 \mu \mathrm{L}$ of PCR-grade water. The primer sequences used are from a previous study [7] and shown in Table 1 . The reaction conditions were denaturation at $95^{\circ} \mathrm{C}$ for $10 \mathrm{~min}$, followed by 40 cycles of $95^{\circ} \mathrm{C}$ for $15 \mathrm{~s}$ and $60^{\circ} \mathrm{C}$ for $1 \mathrm{~min}$. The crossing points of the target genes with $\beta$-actin were calculated using the formula $2^{- \text {(target gene- } \beta \text {-actin) }}$, and relative amounts were quantified.

\section{Evaluation of level of hearing loss}

PTA was performed by measuring air and bone conduction, with hearing calculated as the average of three frequencies $(500,1,000$, and 2,000 Hz). Using the quartering method, the mean PTA value was divided into subgroups of PTA less than $40 \mathrm{~dB}$ and PTA greater than $40 \mathrm{~dB}$.

\section{Statistical analysis}

To investigate the statistical significance of expression of autophagy-related mRNA in the cholesteatoma of CholeOM and the granulation tissue of COM, student's t-test was used for normally distributed data, and Mann-Whitney U test was used for non-normally distributed data. Chi-square test and Fisher's exact test were used in the comparison analysis of demographic data. IBM SPSS version 20 (IBM Corp., Armonk, NY, USA) was used for all statistical analyses. A $p$-value less than 0.05 was considered significant. 


\section{Results}

Demographics and clinical characteristics of subjects

Among the 47 patients with chronic otitis media, 28 were in the COM group and 19 were in the CholeOM group. The COM group contained more bacterial culture-positive pa-

Table 1. Primer sequences for real-time PCR

\begin{tabular}{clc}
\hline Name & \multicolumn{1}{c}{ Sequences } & Size (bp) \\
\hline B-Actin & 5'-GCGAGAAGATGACCCAGATC-3' & 77 \\
& 5'-GGATAGCACAGCCTGGATAG-3' & \\
mTOR & 5'-CCTGCCACTGAGAGATGACA-3' & 168 \\
& 5'-TCCGGCTGCTGTAGCTTATT-3' & \\
P13KC3 & 5'-GGAACACCGACCTCACAGTT-3' & 128 \\
& 5'-CACAGCACCTCCTCTGTGAA-3' & \\
LC3 II & 5'-AGCAGCATCCAACCAAAATC-3' & 187 \\
& 5'-CTGTGTCCGTTCACCAACAG-3' & \\
Beclin-1 & 5'-AGGTTGAGAAAGGCGAGACA-3' & 112 \\
& 5'-AATTGTGAGGACACCCAAGC-3' & \\
Rubicon & 5'-CAGATTCTGCTGCCTCTTCC-3' & 105 \\
& 5'-AGTGTCTGCCCCTCTGAGAA-3' & \\
\hline
\end{tabular}

mTOR: mammalian target of rapamycin, P13KC3: class III phosphatidylinositol 3-kinase, LC3 II: microtubule-associated protein 1A/1B-light chain II, Rubicon: RUN and cysteine rich domain containing beclin 1 interacting protein tients than the CholeOM group (COM vs. CholeOM, 64.3\% vs. $21.1 \%$; $p=0.049$ ). Comparison between the groups showed no difference in age, gender, affected side, type, or degree of hearing loss. Pseudomonas aeruginosa was the most frequently detected strain, followed by methicillin-resistant Staphylococcus aureus (MRSA), coagulase-negative Staphylococcus, Staphylococcus aureus, and Achromobacter xylosoxidans (Table 2).

\section{Expression of mTOR, P13K, LC3 II, Beclin-1, and Rubicon mRNAs}

mTOR, P13KC3, LC3 II, Beclin-1, and Rubicon mRNA was expressed in skin, granulation tissue, and cholematrix obtained from all 47 patients. Expression of $P I 3 K$ mRNA (COM vs. CholeOM, mean \pm SD, $0.009 \pm 0.010$ vs $0.003 \pm 0.004$; $p=$ $0.004)$ was lower and Beclin-1 mRNA $(0.089 \pm 0.107$ vs. $0.176 \pm 0.163 ; p=0.034)$ was higher in the CholeOM group (Table 3).

\section{Expression of $m T O R, P I 3 K, L C 3$ II, Beclin-1, and Rubicon mRNAs in COM and CholeOM patients in the presence of bacteria}

The expression of PI3K mRNA was lower in the CholeOM

Table 2. Demographic and clinical characteristics of patients with chronic otitis media without and with cholesteatoma

\begin{tabular}{|c|c|c|c|}
\hline & $\operatorname{COM}(n=28)$ & CholeOM $(n=19)$ & p-value \\
\hline \multicolumn{4}{|l|}{ Demographics } \\
\hline Age (years) & $55.60 \pm 13.39$ & $48.78 \pm 15.96$ & 0.090 \\
\hline Sex, male:female & $10: 18(35.7: 64.3)$ & $10: 9(52.6: 47.4)$ & 0.508 \\
\hline Disease onset (months) & $9.77 \pm 10.96$ & $8.63 \pm 8.93$ & 0.267 \\
\hline Affected side, Rt:L† & $12: 16(42.9: 57.1)$ & $12: 7(63.2: 36.8)$ & 0.747 \\
\hline \multicolumn{4}{|l|}{ Surgical findings } \\
\hline Culture positive & $18(64.3)$ & $4(21.1)$ & $0.049 *$ \\
\hline Pseudomonas aeruginosa & 5 & 1 & \\
\hline MRSA & 5 & 1 & \\
\hline Staphylococcus aureus & 3 & 0 & \\
\hline CNS & 2 & 1 & \\
\hline Candida & 2 & 0 & \\
\hline Achromobacter xylosoxidans & 0 & 1 & \\
\hline Otorrhea positive & $23(82.1)$ & $12(63.2)$ & 0.136 \\
\hline Revision surgery & $5(17.8)$ & $2(10.5)$ & 0.313 \\
\hline \multicolumn{4}{|l|}{ Audiologic configuration } \\
\hline $\operatorname{PTA}(\mathrm{AC})(\mathrm{dB})$ & $54.35 \pm 21.39$ & $49.60 \pm 20.42$ & 0.581 \\
\hline PTA (BC) (dB) & $28.51 \pm 16.16$ & $22.54 \pm 16.54$ & 0.508 \\
\hline \multicolumn{4}{|l|}{ Hearing loss type } \\
\hline $\mathrm{CHL}$ & $10(35.7)$ & $9(47.3)$ & 0.547 \\
\hline SNHL & $9(32.1)$ & $4(21.0)$ & 0.515 \\
\hline Normal & $9(32.1)$ & $6(31.5)$ & 0.612 \\
\hline
\end{tabular}

Data are presented as number (\%) or mean \pm SD. $* p<0.05$. COM: chronic otitis media without cholesteatoma, CholeOM: chronic otitis media with cholesteatoma, CNS: coagulase-negative staphylococci, PTA: pure tone audiometry, AC: air conduction, $\mathrm{BC}$ : bone conduction, $\mathrm{CHL}$ : conductive hearing loss, SNHL: sensorineural hearing loss 
Table 3. Expression of mRNA encoding autophagy-related proteins in chronic otitis media without and with cholesteatoma

\begin{tabular}{lccc}
\hline Genes & COM $(n=28)$ & CholeOM $(n=19)$ & $p$-value \\
\hline TOR & $0.0009 \pm 0.0005$ & $0.009 \pm 0.106$ & 0.083 \\
P13KC3 & $0.009 \pm 0.010$ & $0.003 \pm 0.004$ & $0.004^{*}$ \\
LC3 II & $0.385 \pm 0.349$ & $0.434 \pm 0.545$ & 0.294 \\
Beclin-1 & $0.089 \pm 0.107$ & $0.176 \pm 0.163$ & $0.034^{*}$ \\
Rubicon & $0.006 \pm 0.005$ & $0.008 \pm 0.010$ & 0.076 \\
\hline
\end{tabular}

Data are presented as mean \pm SD. $* p<0.05$. COM: chronic otitis media without cholesteatoma, CholeOM: chronic otitis media with cholesteatoma, mTOR: mammalian target of rapamycin, P13KC3: class III phosphatidylinositol 3-kinase, LC3 II: microtubule-associated protein 1A/1B-light chain II, Rubicon: RUN and cysteine rich domain containing Beclin 1 interacting protein

Table 4. Expression of mRNA encoding proteins related to autophagy and ER stress in presence of bacteria in patients with chronic otitis media without and with cholesteatoma

\begin{tabular}{lccc}
\hline Genes & COM $(\mathrm{n}=18)$ & CholeOM $(\mathrm{n}=4)$ & p-value \\
\hline mTOR & $0.0009 \pm 0.0005$ & $0.004 \pm 0.008$ & 0.548 \\
PI3KC3 & $0.022 \pm 0.019$ & $0.001 \pm 0.001$ & $0.001^{*}$ \\
LC3 II & $0.380 \pm 0.588$ & $0.155 \pm 0.178$ & 0.484 \\
Beclin- 1 & $0.084 \pm 0.113$ & $0.069 \pm 0.061$ & 0.842 \\
Rubicon & $0.030 \pm 0.061$ & $0.003 \pm 0.005$ & 0.065
\end{tabular}

Data are presented as mean \pm SD. $* p<0.05$. COM: chronic otitis media without cholesteatoma, CholeOM: chronic otitis media with cholesteatoma, mTOR: mammalian target of rapamycin, P13KC3: class III phosphatidylinositol 3-kinase, LC3 II: microtubule-associated protein 1A/1B-light chain II, Rubicon: RUN and cysteine rich domain containing Beclin 1 interacting protein

group than in the COM group when the bacterial culture was positive (COM vs. CholeOM, mean $\pm \mathrm{SD}, 0.022 \pm 0.019$ vs. $0.001 \pm 0.001 ; p=0.001$ ) (Table 4).

\section{Expression of mTOR, PI3K, LC3 II, Beclin-1, and Rubi-} con mRNAs in COM and CholeOM patients in the

\section{presence of otorrhea}

In the presence of otorrhea, the expression of PI3K mRNA was lower in the CholeOM group than in the COM group (COM vs. CholeOM, mean $\pm \mathrm{SD}, 0.049 \pm 0.068$ vs. $0.003 \pm$ $0.004 ; p=0.004$ ) (Table 5).

Expression of $m T O R, P 13 K C 3, L C 3$ II, Beclin-1, FLIP, Rubicon, BIRC2, and BIRC5 mRNAs in patients with over $40 \mathrm{~dB}$ hearing loss

$P I 3 K$ mRNA expression was significantly lower in the CholeOM group than in the COM group when the hearing loss was more than $40 \mathrm{~dB}$ (COM vs. CholeOM, mean $\pm \mathrm{SD}$, $0.083 \pm 0.130$ vs. $0.003 \pm 0.004 ; p=0.005$ ) (Table 6 ).
Table 5. Expression of mRNA encoding proteins related to autophagy and ER stress in presence of otorrhea in patients without and with cholesteatoma

\begin{tabular}{lccc}
\hline Genes & COM $(n=23)$ & CholeOM $(n=12)$ & $p$-value \\
\hline mTOR & $0.0009 \pm 0.0005$ & $0.004 \pm 0.009$ & 0.268 \\
P13KC3 & $0.049 \pm 0.068$ & $0.003 \pm 0.004$ & $0.004^{*}$ \\
LC3 II & $0.557 \pm 1.209$ & $0.468 \pm 0.682$ & 0.842 \\
Beclin-1 & $0.089 \pm 0.121$ & $0.196 \pm 0.202$ & 0.116 \\
Rubicon & $0.040 \pm 0.072$ & $0.009 \pm 0.011$ & 0.156 \\
\hline
\end{tabular}

Data are presented as mean \pm SD. $* p<0.05$. COM: chronic otitis media without cholesteatoma, CholeOM: chronic otitis media with cholesteatoma, mTOR: mammalian target of rapamycin, P13KC3: class III phosphatidylinositol 3-kinase, LC3 II: microtubule-associated protein 1A/1B-light chain II, Rubicon: RUN and cysteine rich domain containing Beclin 1 interacting protein

Table 6. Expression of mRNA encoding autophagy-related proteins in patients with over $40 \mathrm{~dB}$ hearing loss without and with cholesteatoma

\begin{tabular}{lccc}
\hline Genes & COM $(n=19)$ & CholeOM $(n=13)$ & $p$-value \\
\hline mTOR & $0.0006 \pm 0.0002$ & $0.007 \pm 0.015$ & 0.230 \\
PI3KC3 & $0.083 \pm 0.130$ & $0.003 \pm 0.004$ & $0.005^{*}$ \\
LC3 II & $0.298 \pm 0.562$ & $0.201 \pm 0.161$ & 0.809 \\
Beclin- 1 & $0.073 \pm 0.108$ & $0.096 \pm 0.090$ & 0.262 \\
Rubicon & $0.037 \pm 0.072$ & $0.006 \pm 0.007$ & 0.076
\end{tabular}

Data are presented as mean \pm SD. $* p<0.05$. COM: chronic otitis media without cholesteatoma, CholeOM: chronic otitis media with cholesteatoma, mTOR: mammalian target of rapamycin, P13KC3: class III phosphatidylinositol 3-kinase, LC3 II: microtubuleassociated protein 1A/1B-light chain II, Rubicon: RUN and cysteine rich domain containing Beclin 1 interacting protein

\section{Discussion}

Autophagy may be involved in the turnover of essential cytoplasmic components or may be induced by external environmental changes such as depletion of nutrients or pathogen invasion. It is also involved in the differentiation and development of tissues and is reported to play a role in diseases such as cancer, muscular disorders, neurodegeneration, and pathogen infection $[2,8,9]$. Autophagy is associated with cell survival, energy maintenance, and metabolic generation in metabolic diseases, with apoptosis in pulmonary diseases, aggregate clearance and mitochondrial preservation in neurodegenerative diseases and aging, and cell death, chemosensitization, tumor suppression, chemoresistance, radioresistance, and tumor growth in cancers. Autophagy was also shown to be associated with plaque stabilization and regulation of proliferation in vascular diseases and with antigen preservation, pathogen removal, regulation of inflammation, and viral replication in infectious diseases $[8,10,11]$. Therefore, it is likely that autophagy also plays a role in the pathogenesis of chronic otitis media, which is closely related to bacterial infection. 
The autophagy process involves several steps, which are classified somewhat differently by various authors but can be broadly divided into initiation, vesicle nucleation, vesicle elongation, fusion, degradation, and termination $[12,13]$. In the present study, we could not study all of the mRNAs associated with autophagy because the number of cells in the samples collected from of the otitis media cases was insufficient. Therefore, we selected $m$ TOR related to initiation, $P I 3 K$ and Beclin-1 related to vesicle nucleation, LC3 II related to vesicle elongation, and Rubicon related to fusion and degradation. These autophagy-related mRNAs were expressed in both COM and CholeOM groups, even when patients were divided according to detection and non-detection of bacteria, indicating an immune response to bacterial infection. Our results showed that autophagy was involved in all chronic otitis media cases regardless of whether cholesteatoma was present.

The biological role of autophagy is different according to activated or inactivated disease [14-16]. Especially, in pathogen infection, activation of autophagy leads to cellular defense against invasive bacteria and viruses, and inactivation of autophagy allows pathogens to establish a replicative niche. This study did not reveal the role of autophagy in activation and inactivation of COM because we only analyzed the expression patterns of autophagy-related mRNAs in the presence or absence of cholesteatoma and according to clinical manifestations. However, different autophagy-related mRNAs were expressed at different levels between the COM group and CholeOM group. We cannot explain why PI3K mRNA, associated with vesicle nucleation, was expressed at a lower level in the CholeOM group, or why expression of Beclin-1 mRNA was lower in the COM group. Vesicle nucleation during the autophagy process may play an important role in the pathogenesis of chronic otitis media, and even though the same vesicle nucleation process occurs, various autophagy proteins may play different roles depending on the presence of granulation tissue in COM and cholesteatoma in CholeOM.

Activation of phosphoinositide 3-kinase (PI3K) by various factors, such as tumorigenic genes, growth factors, and growth factor receptors, is considered to be a characteristic of cancer. PI3K regulates the development, differentiation, growth, proliferation, and activation of various immune cells that are highly involved in the pathogenesis of innate immune and acquired immune-mediated diseases [17]. However, the role of PI3K in chronic otitis media has not been elucidated. The expression of PI3K in all samples of this study strongly indicated involvement in the pathogenesis of chronic otitis media. Beclin-1 not only activates autophagy, but also inhibits tumorigenesis. Mice with disruption of the Beclin-1 gene have a higher incidence of lymphoma, lung cancer, and liver cancer [17]. A re- cent study also showed that Beclin-1 is associated with the onset and chronicity of otitis media with effusion [7]. Further studies investigating the effects of $P I 3 K$ and Beclin- 1 mRNA expression on the progression, recurrence, chronicity, and degree of hearing loss in otitis media patients are necessary.

Major pathologic findings in the middle ear cavity in chronic otitis media patients include formation of granulation tissue, cholesterol granuloma, cholesteatoma, fibrosis, and changes in bone formation. In this study, the granulation tissue of COM patients was induced by inflammation caused by middle ear exudates and changes in epithelial cells and subepithelial tissue of the middle ear mucosa. Cellular components were abundant in the acute and subacute phases of otitis media, whereas fibrous tissue was abundant in the chronic phase [18-20]. In the CholeOM group, cholesteatoma was characterized by invasive growth of keratinized stratified squamous epithelium into mucosa in the middle ear cavity, destroying the surrounding bone tissue through accumulation of keratin and resulting in more severe and serious complications than in the COM group. Bone destruction involves various enzymes, such as collagenase, acid phosphatase, and acid protease secreted from the inflammatory and granulation tissue, and by the pressure effect of the cholesteatoma itself [18,21-23]. Recently, several types of cytokines secreted from inflammatory cells have been shown to be involved in these actions [24-27]. The results of the present study indicate that autophagy is also involved. The granulation tissues observed in the COM group and the cholesteatoma tissues observed in the CholeOM group induce inflammation in normal tissues, suggesting that the inflammatory response is inevitably related to autophagy gene expression.

The bacterial culture test in chronic otitis media is a necessary test for selecting appropriate antibiotics. In this study, $P$. aeruginosa was detected most frequently in bacterial culture tests of otorrhea, followed by MRSA, coagulase-negative Staphylococcus, S. aureus, and A. xylosoxidans. In both COM and CholeOM groups, complex infections with aerobic and anaerobic bacteria were the most common, with rates as high as $50 \%$. The most common aerobic bacteria were $P$. aeruginosa, and the bacterial culture test results of this study were consistent with those of a previous report [28,29].

In general, the extent of otorrhea in CholeOM is often less than that in non-cholesteatoma otitis, especially in the initial state of epitympanic recess retraction. A large extent of otorrhea indicates that a secondary bacterial infection has occurred. However, in the present study, the detection rate of bacterial growth in pus culture was statistically higher in the CholeOM group than in the COM group. These results suggest that patients in the CholeOM group did not visit the hos- 
pital because the incidence of otorrhea was low; however, it is also possible that, when patients did visit the hospital due to severe otorrhea, the inflammatory reaction induced by bacteria was severe, which indicated that the CholeOM group had a higher positive rate than the COM group. Further studies will be necessary to determine whether PI $3 K$ mRNA expression inhibits the development of otorrhea, whether PI3K is active in the granulation tissues rather than cholesteatoma or otorrhea caused by inflammation of the middle ear, and whether the low expression of PI3K mRNA in the CholeOM group compared with the COM group in the presence of otorrhea and in the culture-positive group is related to vigorous bacterial growth.

Hearing loss is one of the most common symptoms of chronic otitis media. In the present study, the expression of PI3K mRNA was significantly lower in the CholeOM group than in the COM group in the presence of hearing loss greater than $40 \mathrm{~dB}$, suggesting that $P I 3 K$ mRNA may be involved in severe middle ear lesions in the CholeOM group. The degree of hearing impairment is determined by a combination of the size of the tympanic membrane perforation, ossicle conditions, severity of the middle ear inflammation, presence or absence of otorrhea, and inner ear involvement [30]. Therefore, although it is difficult to conclude that the PI $3 K$ expression was related to hearing loss, this association is indirectly supported by the difference in mRNA expression according to the presence of cholesteatoma.

There are some limitations to this study. First, this study was performed only at the mRNA level, and data at the protein level are not available. Second, patients who were classified as the otorrhea-negative group had no otorrhea in the external auditory canal when they visited the hospital, although they had experienced otorrhea in the past. Third, there was a possibility that prophylactic use of antibiotics to reduce inflammation affected bacterial culture and expression of autophagy-related mRNA in both otorrhea-positive and -negative groups. Fourth, we could not elucidate why expression of $P I 3 K$ and Beclin- 1 mRNA was significantly different between $\mathrm{COM}$ and CholeOM groups among the many autophagy-related mRNAs.

In summary, among the various autophagy-related mRNAs studied, expression of PI3K mRNA was significantly lower in the CholeOM group, and that of Beclin-1 mRNA was significantly lower in the COM group, suggesting that different autophagy proteins play important roles in chronic otitis media according to the presence or absence of cholesteatoma.

\section{Acknowledgments}

This work was supported by the National Research Foundation of Korea (NRF) grant funded by the Korea government (NRF-2018 R1A6A1A03025124).

\section{Conflicts of interest}

The authors have no financial conflicts of interest.

\section{Author Contributions}

Conceptualization: Seung Geun Yeo. Data curation: Su Young Jung, Young Il Kim, Sang Hoon Kim. Formal analysis: Su Young Jung, Sang Hoon Kim. Funding acquisition: Sang Hoon Kim, Seung Geun Yeo. Investigation: Seung Geun Yeo. Methodology: Young Il Kim, Seung Geun Yeo. Project administration: Junyang Jung, Su Young Jung. Resources: Young Il Kim, Sang Hoon Kim, Seung Geun Yeo. Software: Su Young Jung, Myung Gu Kim, Young Il Kim. Supervision: Seung Geun Yeo. Validation: Seung Geun Yeo. Visualization: Junyang Jung, Seung Geun Yeo. Writing - original draft: Junyang Jung, Seung Geun Yeo. Writing — review \& editing: Seung Geun Yeo. Approval of final manuscript: all authors.

\section{ORCID iDs}

Junyang Jung

Su Young Jung

Myung Gu Kim

Young Il Kim

Sang Hoon Kim

Seung Geun Yeo

https://orcid.org/0000-0003-3946-5406

https://orcid.org/0000-0001-6398-1297

https://orcid.org/0000-0001-8169-3977

https://orcid.org/0000-0002-2960-1040

https://orcid.org/0000-0001-5045-5060

https://orcid.org/0000-0001-8021-1024

\section{REFERENCES}

1) Długońska H. Autophagy as a universal intracellular process. A comment on the 2016 Nobel Prize in Physiology or Medicine. Ann Parasitol 2017;63:153-7.

2) Fîlfan M, Sandu RE, Zăvăleanu $A D$, GreşiŢă A, Glăvan DG, Olaru DG, et al. Autophagy in aging and disease. Rom J Morphol Embryol 2017;58:27-31.

3) Paparella MM, Schachern PA, Cureoglu S. Chronic silent otitis media. ORL J Otorhinolaryngol Relat Spec 2002;64:65-72.

4) da Costa SS, Paparella MM, Schachern PA, Yoon TH, Kimberley BP. Temporal bone histopathology in chronically infected ears with intact and perforated tympanic membranes. Laryngoscope 1992;102: 1229-36.

5) Paparella MM, Kim CS, Goycoolea MV, Giebink S. Pathogenesis of otitis media. Ann Otol Rhinol Laryngol 1977;86:481-92.

6) Bhutta MF, Thornton RB, Kirkham LS, Kerschner JE, Cheeseman MT. Understanding the aetiology and resolution of chronic otitis media from animal and human studies. Dis Model Mech 2017;10:1289300.

7) Hoon KS, Gu KM, Seon SH, Su KS, Il KY, Geun YS. Lower Beclin-1 mRNA levels in pediatric compared with adult patients with otitis media with effusion. J Int Adv Otol 2018;14:48-52.

8) Choi AM, Ryter SW, Levine B. Autophagy in human health and disease. N Engl J Med 2013;368:651-62.

9) Jiang P, Mizushima N. Autophagy and human diseases. Cell Res 2014;24:69-79.

10) Hsu P, Shi Y. Regulation of autophagy by mitochondrial phospholipids in health and diseases. Biochim Biophys Acta Mol Cell Biol Lipids 2017;1862:114-29.

11) Bento CF, Renna M, Ghislat G, Puri C, Ashkenazi A, Vicinanza M, et al. Mammalian autophagy: how does it work? Annu Rev Biochem 2016;85:685-713.

12) Shintani T, Klionsky DJ. Autophagy in health and disease: a doubleedged sword. Science 2004;306:990-5. 
13) Cuervo AM. Autophagy: in sickness and in health. Trends Cell Biol 2004;14:70-7.

14) Rich KA, Burkett $C$, Webster P. Cytoplasmic bacteria can be targets for autophagy. Cell Microbiol 2003;5:455-68.

15) Dorn BR, Dunn WA Jr, Progulske-Fox A. Bacterial interactions with the autophagic pathway. Cell Microbiol 2002;4:1-10.

16) Coers J, Monahan C, Roy CR. Modulation of phagosome biogenesis by Legionella pneumophila creates an organelle permissive for intracellular growth. Nat Cell Biol 1999;1:451-3.

17) Wechman SL, Pradhan AK, DeSalle R, Das SK, Emdad L, Sarkar D, et al. New insights into beclin-1: evolution and pan-malignancy inhibitor activity. Adv Cancer Res 2018;137:77-114.

18) Abramson M, Gross J. Further studies on a collagenase in middle ear cholesteatoma. Ann Otol Rhinol Laryngol 1971;80:177-85.

19) Kuo CL. Etiopathogenesis of acquired cholesteatoma: prominent theories and recent advances in biomolecular research. Laryngoscope 2015;125:234-40.

20) Olszewska E, Wagner M, Bernal-Sprekelsen M, Ebmeyer J, Dazert $\mathrm{S}$, Hildmann H, et al. Etiopathogenesis of cholesteatoma. Eur Arch Otorhinolaryngol 2004;261:6-24.

21) Blair HC, Kahn AJ, Crouch EC, Jeffrey JJ, Teitelbaum SL. Isolated osteoclasts resorb the organic and inorganic components of bone. $\mathrm{J}$ Cell Biol 1986;102:1164-72.
22) Orisek BS, Chole RA. Pressures exerted by experimental cholesteatomas. Arch Otolaryngol Head Neck Surg 1987;113:386-91.

23) Thomsen J, Jorgensen MB, Bretlau P, Kristensen HK. Bone resorption in chronic otitis media. A histological and ultrastructural study. I. Ossicular necrosis. J Laryngol Otol 1974;88:975-81.

24) Ahn JM, Huang CC, Abramson M. Third place--Resident Basic Science Award 1990. Interleukin 1 causing bone destruction in middle ear cholesteatoma. Otolaryngol Head Neck Surg 1990;103:527-36.

25) Chung JW, Yoon TH. Different production of interleukin-lalpha, interleukin-1beta and interleukin-8 from cholesteatomatous and normal epithelium. Acta Otolaryngol 1998;118:386-91.

26) Schilling V, Negri B, Bujía J, Schulz P, Kastenbauer E. Possible role of interleukin 1 alpha and interleukin 1 beta in the pathogenesis of cholesteatoma of the middle ear. Am J Otol 1992;13:350-5.

27) Chole RA. Cellular and subcellular events of bone resorption in human and experimental cholesteatoma: the role of osteoclasts. Laryngoscope 1984;94:76-95.

28) Erkan M, Aslan T, Sevük E, Güney E. Bacteriology of chronic suppurative otitis media. Ann Otol Rhinol Laryngol 1994;103:771-4.

29) Brook I. Aerobic and anaerobic bacteriology of cholesteatoma. Laryngoscope 1981;91:250-3.

30) Sade J, Berco E, Buyanover D, Brown M. Ossicular damage in chronic middle ear inflammation. Acta Otolaryngol 1981;92:273-83. 